\section{The risk of venipuncture in newborn with severe hemophilia: Case report of a large elbow hemorrhage and literature review of compartment syndrome}

\author{
Giuseppe Lassandro, Anna Amoruso, \\ Valentina Palladino, Viviana Valeria \\ Palmieri, Paola Giordano \\ Pediatric Section, Department of \\ Biomedical Science and Human \\ Oncology, University “Aldo Moro", \\ Bari, Italy
}

\begin{abstract}
Hemophilias are hemorrhagic congenital rare diseases. The gold standard of therapy in hemophilics is the intravenously replacement therapy. We can infuse intravenously plasma derived factors (FVIII for Hemophilia A and FIX for Hemophilia B) or recombinant products (i.e. clotting factor synthetically produced). Venipuncture is not a safe procedure in subjects with hemorrhagic diseases. It is considered an invasive technique with potential massive bleeding and it requires standardized procedures to prevent complications. Local pressure after the procedure (with eventually ice rest) must be always done. In case of bleeding a rapid replacement therapy must be conducted. A severe complication in hemophilia is compartment syndrome. We report a case of massive bleeding in a hemophilic newborn after venipuncture and a literature review of compartment syndrome in hemophiliacs. The aim of this paper is to help physicians in the clinical management to prevent the evolution of a massive bleeding in compartment syndrome.
\end{abstract}

\section{Introduction}

Hemophilias are hemorrhagic congenital rare diseases. The lack, in plasma, of clotting factor VIII (FVIII) defines Hemophilia A instead the lack of clotting factor IX (FIX) causes Hemophilia B. Hemophilia A and B present an X-linked recessive genetic transmission. For this reason, males are sick and females, in the majority of cases, are healthy carriers. ${ }^{1}$ The incidence is respectively 1 per 5000 male births in Hemophilia A and 1 per 30000 male births in Hemophilia B. ${ }^{2,3}$ Hemophilias are categorized according to the plasma concentration of factors in: mild
( 5 to $40 \%$ ), moderate ( 1 to $5 \%$ ) and severe $(<1 \%)$. Severe patients have predominantly spontaneous hemorrhages instead mild patients suffer of bleeding after trauma or surgery. Subjects with moderate hemophilia can exhibit a wide range of symptoms. ${ }^{4}$ The gold standard of therapy in hemophilics is the replacement therapy. Therapy on demand to stop a bleeding or prophylactic regimen to prevent hemorrhages. We can infuse intravenously plasma derived factors (FVIII for Hemophilia A and FIX for Hemophilia B) or recombinant products (i.e. clotting factor synthetically produced by pharmaceutical industry). 5,6 In hemophilic patients the development of inhibitors is a possible complication related to the therapy. Inhibitors are antibodies against the clotting factors infused. In last years was approved an innovative molecule. Emicizumab, the first of the non $\square$ factor therapies to be licensed for patients with inhibitor, is a bispecific antibody that binds to both factor IXa (FIXa) and factor X (FX) and in doing so supports conformational changes that allows FIXa to activate FX in the absence of FVIII. Emicizumab may prevent hemorrhages in hemophilic people with inhibitiors. ${ }^{7}$ The diagnosis of hemophilia is suspected in childhood for a positive family history or for the appearance of an early bleeding without a known cause. ${ }^{8}$ Large hemorrhages may occur in infants with hemophilia in injection sites for recurrent micro bleedings due to the difficult to find an appropriate vein but, also, for the immature fragile vascular structure. Venipuncture in newborn with severe hemophilia is not a safe procedure. International guidelines for the management of hemophilia suggest to use small butterfly needles (23- or 25-gauges), avoid surgical techniques to isolate a vein (except in emergency) and apply pressure for 3-5 min after venipuncture. ${ }^{1}$ Uncontrolled venipuncture hemorrhage can be the cause of a serious complication: the compartment syndrome. ${ }^{9}$ In literature there are limited experiences of acute compartment syndrome (ACS) in hemophilic subjects. In this paper, we describe a newborn with severe hemophilia A who presented a serious volar arm bleeding post venipuncture. The early detection of the problem and the intensive treatment with recombinant FVIII could prevent the compartment syndrome.

\section{Case Report}

We report a case of a male newborn by Polish mother with family history of severe hemophilia (two maternal uncles). He was
Correspondence: Paola Giordano, Department of Biomedical Science and Human Oncology, University of Bari "Aldo Moro", Piazza Giulio Cesare 11, 70124 Bari, Italy.

Tel.: +39.0805592287.

E-mail: paola.giordano@uniba.it

Key words: Newborn, hemophilia, venipuncture, bleeding, compartment syndrome.

Contributions: All authors contributed equally.

Conflict of interest: The authors declare no conflict of interest

Funding: None.

Ethical approval: This article does not contain any studies with animals performed by any of the authors. All procedures performed in studies involving human participants were in accordance with the ethical standards of the institutional and/or national research committee and with the 1964 Helsinki declaration and its later amendments or comparable ethical standards.

Informed consent: Informed consent was obtained from parents of participant included in the study.

Received for publication: 26 September 2020. Accepted for publication: 26 February 2021.

This work is licensed under a Creative Commons Attribution-NonCommercial 4.0 International License (CC BY-NC 4.0).

${ }^{\circ}$ Copyright: the Author(s), 2021

Licensee PAGEPress, Italy

Hematology Reports 2021; 13:8967

doi:10.4081/hr.2021.8967

born from vaginal delivery at 40 weeks after a pregnancy uncomplicated. No data was available on clinical status of two mother's brothers and on inhibitor appearance because she had lost contacts with her polish family. Physical examination of newborn was negative, no traumatic lesions related at delivery were detected. He was discharged on the third day with recommendation to contact regional treatment center for pediatric hemophilia (PHTC). After seven days his mother noted several bruises in the thoracic area of the infant. Parents carried him in a peripheral hospital without hemophilia section. In emergency room, in the suspicion of coagulation disorder, a venipuncture for blood exams was performed. The complete blood count was normal but coagulation studies revealed an isolated prolonged activated partial thromboplastin time. To investigate these results 
baby has been subjected at another venipuncture. Coagulation factors (VIII, IX, XI) and Von Willebrand study (antigen and activity) were measured. The detection of a very low plasma factor VIII level $(<1 \%)$ confirmed the diagnosis of severe hemophilia A. Doctor of emergency room reaffirmed the indication at parents to contact as soon as possible a PHTC. Newborn was conducted after three days at PHTC. Here at the first observation he presented a swollen with vast ecchymosis on his right arm (Figure 1). He was hospitalized. Ultrasound investigation revealed a large muscle hematoma $(45 \times 39 \mathrm{~mm})$ with signs of skin suffering. The Doppler study showed no alteration in the blood flow of the vessels. Patient was immediately treated with recombinant factor VIII for seven successive days. The hematoma was significantly reduced, necrotic areas of the skin were not recorded and the ultrasound examination never showed vascular pain. At discharge he started a prophylaxis program with recombinant FVIII three times a week. After two weeks he returned to the hospital for the physical examination and blood tests. The exam highlighted further clinical improvement without sequelae but blood exam revealed the appearance of inhibitor against factor VIII (100 BU). Prophylaxis was stopped for the inefficacy of replacement therapy. At last follow up (six months later) persisted inhibitor (89 BU) and the arm showed no injury (Figure 2). Doctors of PHTC proposed at parents to start prophylaxis, subcutaneously, with Emicizumab to protect against possible bleedings.

\section{Discussion}

The bleeding tendency associated with hemophilia $\mathrm{A}$ is proportional to the degree of factor VIII deficiency. Subjects with factor VIII level plasma inferior to $1 \%$ are affected by severe hemophilia A. They present spontaneous and traumatic (also for surgery) hemorrhages. ${ }^{1}$ Intra-articular bleedings (prevalently knee, ankle and elbow) are the typical locations of events in people with hemophilia. Repeated hemarthroses lead to joint damage. The main complication of hemophilic is, indeed, the chronic arthropathy. Arthropathy causes pain, functional impotence and in the final stages motor disability. ${ }^{10,11}$ Replacement therapy with factor VIII (plasma-derived or recombinant) stops bleeding. Periodic intravenous infusion (two or three times a week) depending on the therapeutic regimen according to drug used to prevent spontaneous hemorrhages and, so, it reduces the risk of arthropathy. Children with hemophilia received precociously these prophylactic therapeutic approaches (before two years old and after the first articular bleeding). ${ }^{12}$ Therefore veins must be treated with care, they are the lifelines for a person with hemophilia. Another complication of hemophilic subjects is the appearance of the inhibitor. Inhibitors are neutralizing antibodies against infused factor VIII. The development of them occurs in up to $33 \%$ of patients with severe hemophilia A, in $13 \%$ of those with non-severe hemophilia A. The cause of the development of inhibitors is related to genetic risk (type of mutations and family history of inhibitors) or nongenetic risk (intensive treatment at the first factor VIII exposure and probably the type of drugs). ${ }^{13}$ In the presence of an inhibitor (for the inefficacy of the replacement therapy) the risk of major morbidity and the cost of care increase substantially. Consequences of bleeding and the demands of treatment increase the disease burden on patients and their families, leading to reduced quality of life, financial stress, and strained relationships. ${ }^{14}$ Challenges in hemophilic newborns are different from those in older children and adults. In the newborn period are common intracranial hemorrhages for difficult deliveries, genital dripping for ritual circumcision and, mostly, venipuncture bleedings. Awareness of signs and symptoms and the treatment of complications play a key role in preparing appropriate care plans and implementing preventive programs. ${ }^{15}$ The management of hemophilic neonates must be under the strict surveillance of the hemophilic reference center. Physicians and nurse, expert in the field, may prevent events life-threatening. Venipunctures and other invasive procedures must be reduced o postponed if not necessary. Pricking a vein can be difficult due to the anatomical characteristics of the newborn and repeated attempts can cause

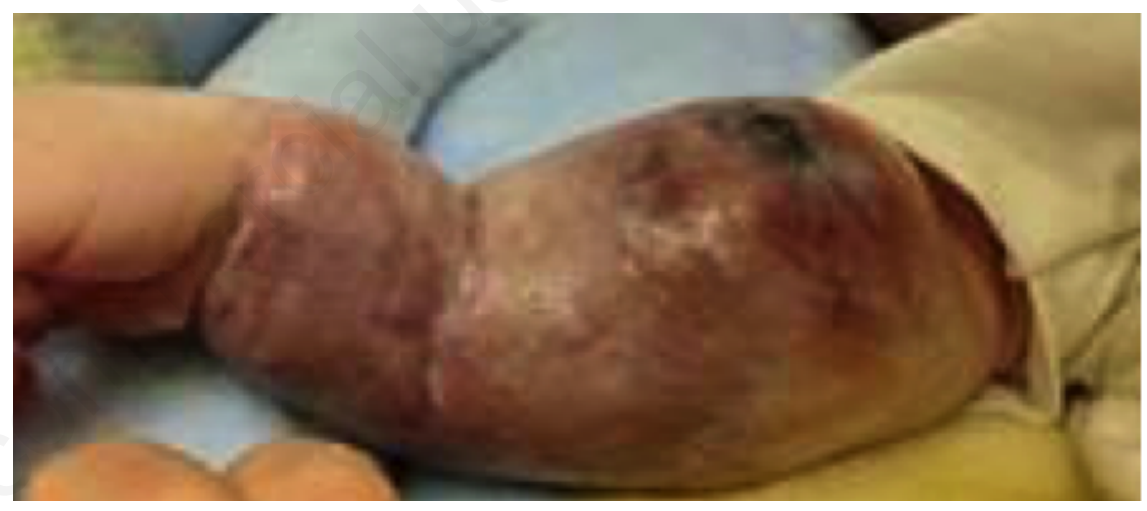

Figure 1. First clinical observation of the right arm of the newborn.

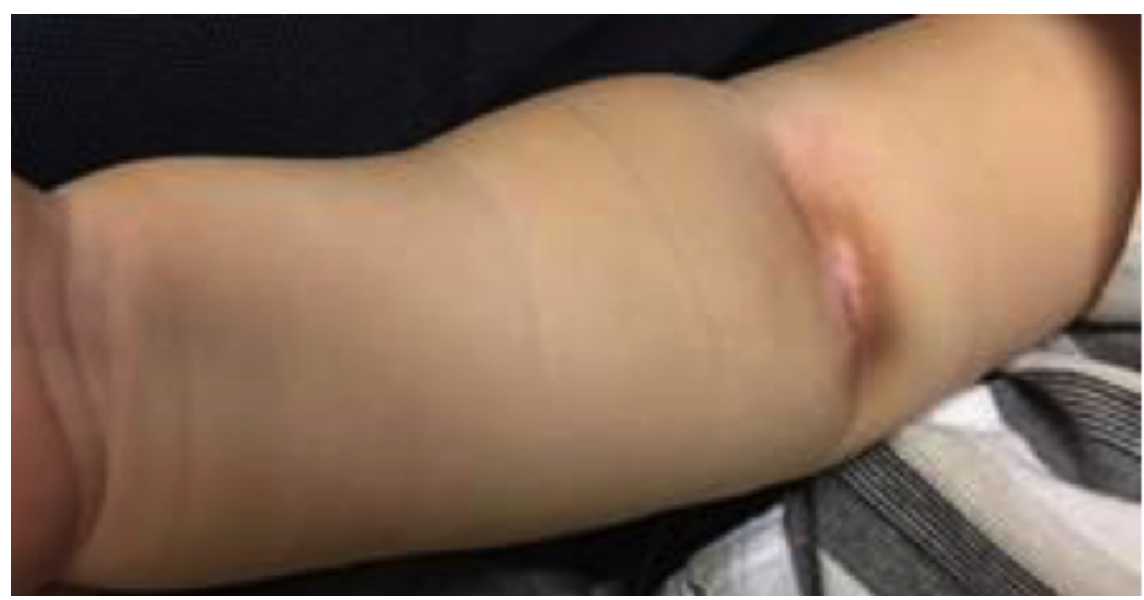

Figure 2. Patient's right arm at follow-up performed six months later. 
micro-lesions in the surrounding tissues that can bleed. ${ }^{16}$ Guidelines suggest, always, to perform a local compression after the procedure even if is not evident a bleeding in progress. The application of ice before and after venipuncture (as recommended during vaccination practice) can be useful to determine vasoconstriction that reduces the risk of bleeding. ${ }^{17}$ The clinical surveillance must be ensure for at least 24-48 hours. At the first sign of bleeding, the replacement therapy with factor VIII must be acted promptly (eventually repeated after 12-24 hours). Sometimes hospitalization, due to the fragility of neonatal clinical conditions, can be helpful to obtain a stable venous access for therapies (avoiding more venipunctures) and to guarantee surveillance. If the bleeding is not immediately controlled, acute compartment syndrome may occur. ACS is a rare but potentially devastating complication after venipuncture in people with hemophilia. ${ }^{18}$ Uncontrolled hemorrhage may generate a critical intersti- tial pressure in the closed osseo-fascial compartment. The elevation of pressure causes microvascular compromise and a reduction in the perfusion gradient. This leads to ischaemia of the tissue within that compartment. ACS is considered a surgical emergency that requires timely evaluation and treatment. The first step in the management of a suspected compartment syndrome should be sufficient the substitution of clotting factors, which may help lower the compartment pressure. A late intervention of ACS develops irreversible tissue ischaemia with potentially disastrous neurological deficits, muscle necrosis, ischaemic contracture, infection, chronic pain and finally amputation and even death. The treatment of ACS is surgical: quickly removal of all circumferential dressings down to the skin, usually followed by open fasciotomy. There are no specific pre-operative guidelines for factor replacement levels during fasciotomy, but is appropriate to maintain a plasma factor VIII level around $100 \%$ as in all major surgery. Infection is a possible complication that can aggravate blood loss. ${ }^{9}$ There are limited experiences in literature of ACS in hemophilic subjects (Table 1). In 1977 Lancourt et al. observed, in 200 hemophilics, 34 episodes of bleeding into the hand and forearm. Fasciotomy was not performed in any of them but in six episodes of bleeding into the anterior muscles of the forearm was complicated by contracture, neuropathy, or both. For authors an early diagnosis with intensive replacement of the missing clotting factor was essential to avoid an irreversible damage. ${ }^{19}$ In 1989 Nixon et al. described a case of ACS post venipuncture in the arm in a three-monthold male infant with fever and irritability. The lack of a positive family history and the unusual clinical presentation of the patient had delayed the diagnosis of hemophilia. In the case described, ACS resolved with only clotting factor replacement without surgical decompression. ${ }^{20}$ In 1994 Dumontier et al. focused the evolution of ACS in 12 cases of

Table 1. Acute compartment syndrome in hemophilics.

\begin{tabular}{|c|c|c|c|c|c|}
\hline Study, year & Population & Injury & Anatomic location & Management & Outcome \\
\hline Lancourt, $1977^{19}$ & $\begin{array}{l}200 \text { Hemophilics, } \\
34 \text { cases of bleeding }\end{array}$ & $\begin{array}{l}\text { Unknown } \\
\text { cases bleeding }\end{array}$ & Hand and forearm & No fasciotomy & $\begin{array}{l}\text { Complications in six } \\
\text { (contracture, } \\
\text { neuropathy or both) }\end{array}$ \\
\hline Nixon, $1989^{20}$ & $\begin{array}{l}1 \text { case of ACS in } \\
\text { hemophilia }\end{array}$ & After venipunture & Forearm & $\begin{array}{l}\text { Clotting factor } \\
\text { replacement; no } \\
\text { fasciotomy }\end{array}$ & Resolved \\
\hline Dumontier, $1994^{21}$ & $\begin{array}{l}12 \text { cases of ACS in } \\
\text { hemophilics }\end{array}$ & $\begin{array}{l}3 \text { minor trauma; } \\
9 \text { no trauma }\end{array}$ & $\begin{array}{l}10 \text { cases in forearm; } \\
2 \text { cases in hand }\end{array}$ & $\begin{array}{l}\text { Clotting factor } \\
\text { replacement; } 1 \text { case } \\
\text { faciotomy and clot } \\
\text { removal on the } 11^{\text {th }} \text { day; } \\
1 \text { case fasciotomy on } \\
\text { the } 3^{\text {rd }} \text { day, clot } \\
\text { removal plus skin } \\
\text { grafting on the } 9^{\text {th }} \text { day }\end{array}$ & $\begin{array}{l}\text { Sequelae and } \\
\text { incomplete recovery in } \\
\text { two cases }\end{array}$ \\
\hline $\begin{array}{l}\text { Rodriguez- } \\
\text { Merchan, } 2013^{21}\end{array}$ & $\begin{array}{l}3 \text { cases of ACS in } \\
\text { hemophilics }\end{array}$ & $\begin{array}{l}1 \text { Ulnar claw; } 1 \text { forearm } \\
\text { contusion; } 1 \text { distal radius }\end{array}$ & Upper limb & $\begin{array}{l}\text { Clotting fracture } \\
\text { factor replacement; } \\
\text { No fasciotomy }\end{array}$ & $\begin{array}{l}\text { A case of irreversible } \\
\text { damage }\end{array}$ \\
\hline Watts, $2005^{22}$ & $\begin{array}{l}1 \text { case of ACS in severe } \\
\text { hemophilia A and inhibitor }\end{array}$ & After trauma & Forearm & $\begin{array}{l}\text { Fasciotomy, blood } \\
\text { transfusion recombinant } \\
\text { factor VIIa }\end{array}$ & Resolved \\
\hline Kim, $2013^{22}$ & 1 case of ACS in hemophilia A & No trauma & Volar forearm & $\begin{array}{l}\text { Clotting factor replacement; } \\
\text { Fasciotomy }\end{array}$ & Resolved \\
\hline Abdelhalim, $2015^{23}$ & $\begin{array}{l}1 \text { case of ACS in severe } \\
\text { hemophilia A and inhibitor }\end{array}$ & $\begin{array}{l}\text { No trauma, } \\
\text { Strenuous exercise }\end{array}$ & Bilateral forearms & $\begin{array}{l}\text { Clotting factor replacement; } \\
\text { decompression; fresh } \\
\text { frozen plasma; factor VIII } \\
\text { inhibitor bypassing activity; } \\
\text { skin graft }\end{array}$ & Resolved \\
\hline Niblock, $2016^{24}$ & $\begin{array}{l}2 \text { cases of ACS in mild } \\
\text { hemophilia B and A }\end{array}$ & Post trauma & Thigh & Clotting factor replacement & Resolved \\
\hline Jones, $2013^{25}$ & $\begin{array}{l}1 \text { case of ACS in mild } \\
\text { hemophilia B }\end{array}$ & Contusion & Thigh & $\begin{array}{l}\text { Fasciotomy; blood transfusion; } \\
\text { plastic surgeons; skin graft }\end{array}$ & Resolved \\
\hline Reynolds, $2017^{26}$ & $\begin{array}{l}1 \text { case of ACS in mild } \\
\text { hemophilia B }\end{array}$ & $\begin{array}{l}\text { Olecranon } \\
\text { fracture }\end{array}$ & Forearm & $\begin{array}{l}\text { Clotting factor replacement; } \\
\text { fasciotomy }\end{array}$ & Resolved \\
\hline Baghdadi, $2019^{27}$ & $\begin{array}{l}1 \text { case of ACS in severe } \\
\text { hemophilia A }\end{array}$ & Post synovectomy & knee & $\begin{array}{l}\text { Clotting factor replacement; } \\
\text { corticosteroids; fasciotomy }\end{array}$ & $\begin{array}{l}\text { Resolved, no sensory } \\
\text { or motor sequelae }\end{array}$ \\
\hline
\end{tabular}

ACS, acute compartment syndrome. 
hemophilics with hemorrhage in the upper limb. They, again, confirmed the importance of clotting factor replacement as first line therapy because fasciotomy and sequelae were linked with late diagnosis and therapy. ${ }^{21}$ In 2005 Watts reported a case of ACS in forearm after trauma in a patient with severe hemophilia A and high-titer factor VIII inhibitor. An emergency fasciotomy was performed and then bleeding was controlled with recombinant factor VIIa. Fasciotomy for compartment syndrome is safe in hemophilic patients with inhibitors, but only in experienced hands and with coordination between the surgeon and hematologist. ${ }^{22}$ Rodriguez-Merchan reported, in his experience of three cases with ACS until 2013, a case of irreversible damage. The author stressed the importance of an expert medical consultation because in his worst case ACS had likely started 4 days prior to seeking medical attention. ${ }^{23}$ In the same year Kim et al. reported a single case successful resolved with fasciotomy. ${ }^{24}$ In 2015 Abdelhalim et al. recorded a bilateral ACS due to hemorrhage for strenuous exercise. The easy tendency to bleeding is related to the presence of inhibitor and, obviously, to lack of prophylaxis with factor VIII. ${ }^{25}$ In 2016 Niblock et al. reported two cases of ACS as the first clinical sign to suspect hemophilia. The author reported subjects with mild hemophilia. They, as above written, can have severe bleeding only after trauma. ${ }^{26}$ This consideration, but for mild hemophilia B, was yet updated by Jones and Reynolds in two different papers. ${ }^{27,28}$ In 2019 Baghdadi S et al. reported an ACS in hemophilic after knee synovectomy. Synovectomy is a procedure to contrast chronic arthropathy. This report remarked two concepts: the importance of hemostatic coverage before and after surgery and the tissue fragility during arthropathy. ${ }^{29}$

\section{Conclusions}

Compartment syndrome is luckily an uncommon complication in hemophilic patients. It occurs when a bleeding determines a critical increase in blood pressure within a confined osseo-fascial compartment. The subsequent decline in perfusion pressure can lead to irreversible tissue damage and necrosis. The first line therapy is the replacement therapy and, if there is no rapid improvement, a surgical fasciotomy is required. Our case in newborn with a positive outcome teaches some considerations. First of all, venipuncture is considered an invasive procedure with potential massive bleeding. Local pressure after the procedure (with eventually ice rest) must be always done. In case of bleeding a rapid replacement therapy must be conducted. The PHTC should be contacted to monitor the clinical evolution. In case of inhibitors, new non-substitutive approaches are available and should take in account to prevent bleeding. Hemophilic children and their caregiver must be accompanied on the treatment path and never abandoned to improve social quality of life and to reduce economic impact. ${ }^{30,31}$

\section{References}

1. Srivastava A, Brewer AK, MauserBunschoten EP, et al. Guidelines for the management of hemophilia. Haemophilia 2013;19:e1-47.

2. Stonebraker JS, Bolton-Maggs $\mathrm{PH}$, Soucie JM, et al. A study of variations in the reported haemophilia A prevalence around the world. Haemophilia 2010;16:20-32.

3. Stonebraker JS, Bolton-Maggs PH, Soucie JM, et al. A study of variations in the reported haemophilia B prevalence around the world. Haemophilia 2012;18:e91-4.

4. Ljung R, Petrini P, Nilsson IM. Diagnostic symptoms of severe and moderate haemophilia A and B. A survey of 140 cases. Acta Paediatr Scand 1990;79:196-200.

5. Rocino A, Coppola A, Franchini M, et al. Principles of treatment and update of recommendations for the management of haemophilia and congenital bleeding disorders in Italy. Italian Association of Haemophilia Centres (AICE) Working Party. Blood Transfus 2014;12:575-98.

6. Schiavoni M, Napolitano M, Giuffrida G, et al. Status of Recombinant Factor VIII Concentrate Treatment for Hemophilia a in Italy: Characteristics and Clinical Benefits. Front Med (Lausanne) 2019;6:261.

7. Oldenburg J, Mahlangu JN, Kim B, et al. Emicizumab Prophylaxis in Hemophilia A with Inhibitors. N Engl J Med 2017;377:809-18.

8. Giordano P, Franchini M, Lassandro G, et al. Issues in pediatric haemophilia care. Ital J Pediatr 2013;39:24.

9. Donaldson J, Goddard N. Compartment Syndrome in Patients With Haemophilia. J Orthop 2015;12:237-41.

10. Nacca CR, Harris AP, Tuttle JR. Hemophilic Arthropathy. Orthopedics 2017;40:e940-e6.

11. Giordano P, Brunetti G, Lassandro G, et al. High serum sclerostin levels in chil- dren with haemophilia A. $\mathrm{Br}$ J Haematol 2016;172:293-5.

12. Castaman G, Linari S. Prophylactic versus on-demand treatments for hemophilia: advantages and drawbacks. Exp Rev Hematol 2018;11:567-76.

13. Peyvandi F, Makris M. Inhibitor development in haemophilia. Haemophilia 2017;23:3.

14. Giordano P, Lassandro G, Valente M, et al. Current management of the hemophilic child: a demanding interlocutor. Quality of life and adequate cost-efficacy analysis. Pediatr Hematol Oncol 2014;31:687-702.

15. Arcieri R, Molinari AC, Farace S, et al. Uncovered needs in the management of inherited bleeding disorders in Italy. Blood Transfus 2014;12:s563-6.

16. Economou M, Banov, L, Ljung R. Perinatal aspects of haemophilia. Eur J Haematol Suppl 2014;76:21-5.

17. Santagostino E, Riva A, Cesaro S, et al. Consensus statements on vaccination in patients with haemophilia-Results from the Italian haemophilia and vaccinations (HEVA) project. Haemophilia 2019;25:656-67.

18. Lyu J, Wu W, Xiang Z, Huang F. Large hemorrhage due to venipuncture in the elbow of a patient with severe hemophilia: A case report and literature review. Exp Ther Med 2016;11:1023-6.

19. Lancourt JE, Gilbert MS, Posner MA. Management of bleeding and associated complications of haemophilia in the hand and forearm. J Bone Joint Surg Am 1977;59:451-60.

20. Nixon RG, Brindley GW. Hemophilia presenting as compartment syndrome in the arm following venipuncture. A case report and review of the literature. Clin Orthop Relat Res 1989;244:176-81.

21. Dumontie C, Sautet A, Man M, et al. Entrapment and compartment syndromes of the upper limb in haemophilia. J Hand Surg Br 1994;19:427-9.

22. Watts RG. Successful use of recombinant factor VIIa for emergency fasciotomy in a patient with hemophilia A and high titer inhibitor unresponsive to factor VIII inhibitor bypassing activity. Am J Hematol 2005;79:58-60.

23. Rodriguez-Meerchan EC. Acute compartment syndrome in haemophilia. Blood Coagul Fibrinolysis 2013;24:677-82.

24. Kim J, Zelken J, Sacks JM. Spontaneous Forearm Compartment Syndrome in a Boy With Hemophilia A: A Therapeutic Dilemma. Eplasty 2013;13:e16.

25. Abdelhalim MA, Shaw CR, Al-Rub $Z A$, et al. Bilateral Upper Limb 
Compartment Syndrome Induced by Strenuous Exercise in a Patient With Haemophilia A and a Low Titre Inhibitor. Haemophilia 2015;21:e517-9.

26. Niblock A, Donnelly K, Sayers F, et al. Avoidable Compartment Syndrome! High Index of Suspicion for a Newly Presenting Haemophiliac: A Case Series. Case Rep Emerg Med 2016;2016:3263261.

27. Jones G, Thompson K, Johnson M. Acute compartment syndrome after minor trauma in a patient with undiagnosed mild haemophilia B. Lancet 2013;382:1678.

28. Reynolds JM, Christophersen C, Mulcahey MK. Acute Compartment Syndrome After an Olecranon Fracture in a Patient With Mild Hemophilia B. J Orthop Case Rep 2017;7:98-101.

29. Baghdadi S, Baghdadi T, Ayati Firoozabadi $M$, et al. Acute Compartment Syndrome After Knee Synovectomy in a Patient With
Hemophilia A: A Case Report. JBJS Case Connect 2019;9:e0402.

30. Negri L, Buzzi A, Aru AB, et al. Perceived well-being and mental health in haemophilia. Psychol Health Med 2020;26:1-11.

31. Valente M, Cortesi PA, Lassandro G, et al. Health economic models in hemophilia A and utility assumptions from a clinician's perspective. Pediatr Blood Cancer 2015;62:1826-31. 